\title{
Novel mutations and the ophthalmologic characters in Chinese patients with Wolfram Syndrome
}

\author{
Youjia Zhang ${ }^{1}$, Lili Feng ${ }^{1}$, Xiangmei Kong ${ }^{1}$, Jihong $\mathrm{Wu}^{1,2}$, Yuhong Chen ${ }^{1,2,3,4^{*}}$ (D) and Guohong Tian ${ }^{1,2,3,4^{*}}$
}

\begin{abstract}
Background: Wolfram Syndrome (WFS) is a rare autosomal recessive neurodegenerative disease which has a wide spectrum of manifestations including diabetes insipidus, diabetes mellitus, optic atrophy and deafness. WFS1 and CISD2 are two main causing genes of WFS. The aim of this study was to illustrate the ophthalmologic manifestations and determine the genotype of Chinese WFS patients.

Results: Completed ophthalmic examinations and family investigations were performed on 4 clinically diagnosed WFS patients from 4 unrelated families. Genetic testing was done by the next generation sequencing of candidate genes. One patient carried a homozygous mutation (c.272_273del) in CISD2, two patients carried compound heterozygous mutations (c.1618 T > G + c.2020G > A and c.1048T > A + c.2020G > A) in WFS1, and one patient carried a heterozygous mutation (c.937C > T) in WFS1. Three of them were novel mutations.

Conclusions: Our study indicated WFS in Chinese is a neurodegenerative disease with both wide spectrum of clinical features and genetic heterogeneity. We found three novel mutations in WFS patients, and to our best knowledge, this is the first report of Chinese WFS patient with mutation in CISD2.
\end{abstract}

Keywords: Wolfram syndrome, DIDMOAD, Optic atrophy, Next generation sequence, WFS1, CISD2

\section{Background}

Wolfram syndrome (WFS; MIM \#222300), first described in 1938 by Wolfram and Wagener, is a rare hereditary autosomal recessive disease. The prevalence of WFS was estimated to be 1 in 770,000 in UK [1] and 1 in 710,000 in the Japanese population [2]. As a progressive neurodegenerative disorder, WFS has a wide spectrum of clinical manifestations. The main phenotypes of WFS are diabetes insipidus (DI), diabetes mellitus (DM), optic atrophy (OA) and deafness (D) [1,3]. Around 50\% patients harbor all these manifestations, so WFS was also referred to as the acronym DIDMOAD syndrome [1, 3, 4]. Other common manifestations include neurologic and psychiatric disorders, renal tract abnormalities, endocrine disorders, as well as many others [5]. The major diagnosis criterion of WFS is the coincidence of early onset type $1 \mathrm{DM}$ and

\footnotetext{
* Correspondence: yuhongchen@fudan.edu.cn; valentian99@hotmail.com ${ }^{1}$ Department of Ophthalmology and Visual Science, Eye, Ear, Nose and Throat Hospital, Shanghai Medical College, Fudan University, Shanghai, China, 83 Fenyang Road, Shanghai 200031, China

Full list of author information is available at the end of the article
}

bilateral OA before the second decade [1,5-7]. Because of the multi-system neurodegeneration, the prognosis of WFS is very poor and the patients' median life expectancy is about 30 years (range $25-49$ years) [1].

WFS1 on chromosome 4 is the causative gene of Wolfram Syndrome type 1 (WFS1) [8], and the loss-offunction mutations of WFS1 have been identified in most of patients with WFS [5, 9]. WFS1 encodes wolframin, an endoplasmic reticulum (ER) transmembrane protein [10]. Wolframin is widely expressed in neurons, pancreas, heart, muscle, liver, spleen and kidney [11]. It has also been detected in optic nerve glial cells and retinal ganglion cells $[12,13]$. The main function of wolframin are reducing the ER stress, maintaining the $\mathrm{Ca}^{2+}$ homeostasis and regulating the biosynthesis and secretion of insulin [14-16].

In addition, mutations of CISD2 are responsible for Wolfram Syndrome type 2 (WFS2; MIM \#604928), which has variant features including gastrointestinal ulceration and bleeding tendency without diabetes insipidus [17-19]. CISD2, CDGSH iron-sulfur domain-containing protein 2, located on chromosome 4q22-24, encodes endoplasmic 


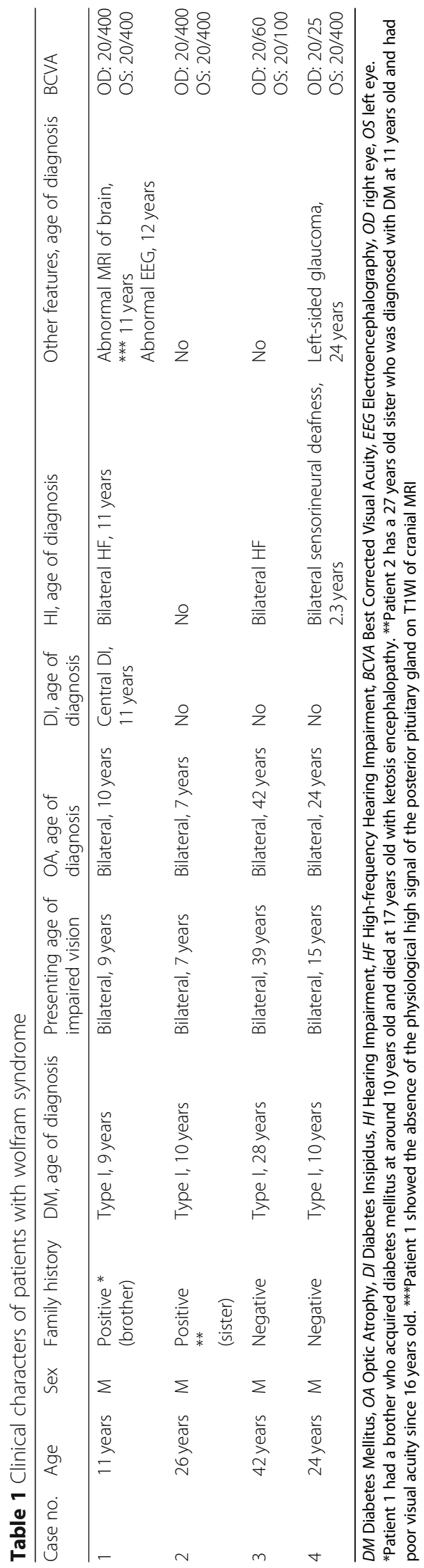




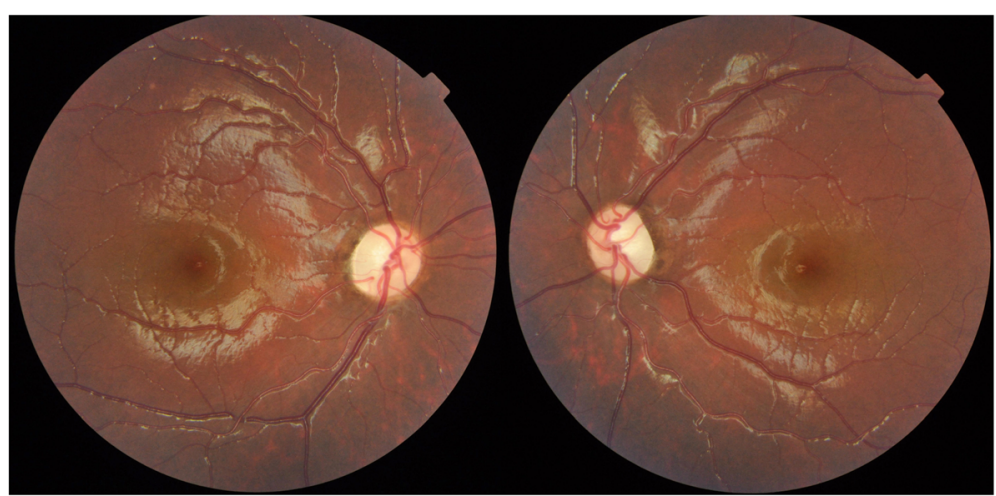

Fig. 1 Fundus photography of the patient with CISD2 mutation. Fundus photography shows optic disc diffused pallid bilateral without diabetes retinopathy

reticulum intermembrane small protein (ERISP) [19]. Although the biological functions of CISD2 still remain incompletely defined, some studies show that it has a similar role with WFS1 in maintaining the homeostasis of $\mathrm{Ca}^{2+}$ and ER and the cross-talk between ER and mitochondria [20, 21].

In this study, we performed a clinical and genetic investigation on 4 unrelated Chinese patients with WFS. We systematically reviewed their clinical ophthalmologic features and identified 3 novel mutations in WFS1 and CISD2 gene. And we reported the first Chinese patient with WFS2 carried a homozygous mutation in CISD2.

\section{Patients and methods}

\section{Patients}

We retrospectively reviewed 4 consecutive patients diagnosed with WFS at Ophthalmology Department of Eye Ear Nose and Throat Hospital of Fudan University from 2013 to 2018. This study was approved by the Eye Ear Nose and Throat Hospital of Fudan University Institutional Review Board, and written formal consent was obtained from all enrolled patients or their legal guardians. Patients were enrolled in our study when meeting one of the following two criteria: 1) the early onset DM and progressive OA, not explained by any other diseases; 2) the identification of 2 pathological WFS1/CISD2 mutations. DM was diagnosed by WHO criteria [22]. OA was confirmed by funduscopic examination of the optic nerve head with pallid appearance and by the evidence of atrophy of the peripapillary nerve fiber layer on optical coherence tomography (OCT). Magnetic resonance imaging (MRI) or computed tomography (CT) scan was also utilized to exclude compressive optic neuropathies. 110 healthy Chinese people, without diagnosis of DM, OA or any other serious ocular or systematic diseases, were also included in this study.

\section{Clinical investigation}

All patients underwent a complete ophthalmologic examination, including visual acuity (VA) examination, intraocular pressure measurement, slit-lamp biomicroscopy, ophthalmoscope, visual fields assessment (Carl Zeiss

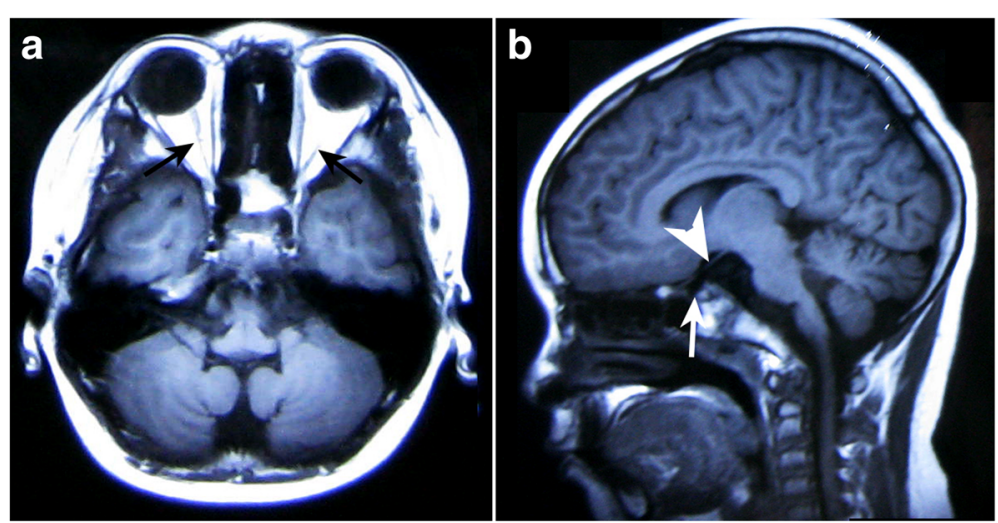

Fig. 2 The magnetic resonance imaging (MRI) of the patient with CISD2 mutation. a: axial T1WI shows bilateral atrophy of optic nerves (black arrows) and (b): sagittal T1WI shows absence of the physiological high signal of the posterior lobe of the pituitary gland (white arrow). The arrow head shows the thinning optic nerves 
Meditec, Inc., Dublin, CA, United States), electroretinography (ERG) and visual evoked potentials (VEP) (LKC UTAS E3000 LKC Technologies, Inc., United States). The OCT (Cirrus OCT 5000, Carl Zeiss Meditec, Inc., Dublin, CA, United States) was performed for each patient to evaluate retinal nerve fiber layer (RNFL) thickness. The MRI was performed in 2 patients and CT scan was completed in the other 2 patients. The audiological, urological, neurological and psychiatric examinations results were recorded from the medical records.

\section{Genetic analysis}

Genomic DNA samples were extracted from whole blood samples of the patients, their relatives, and 110 healthy Chinese people. Genetic testing was performed in all four patients by next generation sequence (NGS). A panel including 790 ophthalmology associated genes were sequenced by Illumina HiSeq 2000 (Illumina, Inc., San Diego, CA, United States) sequencing system. The average depth was 200x. Family members of the probands were validated by Sanger sequence.

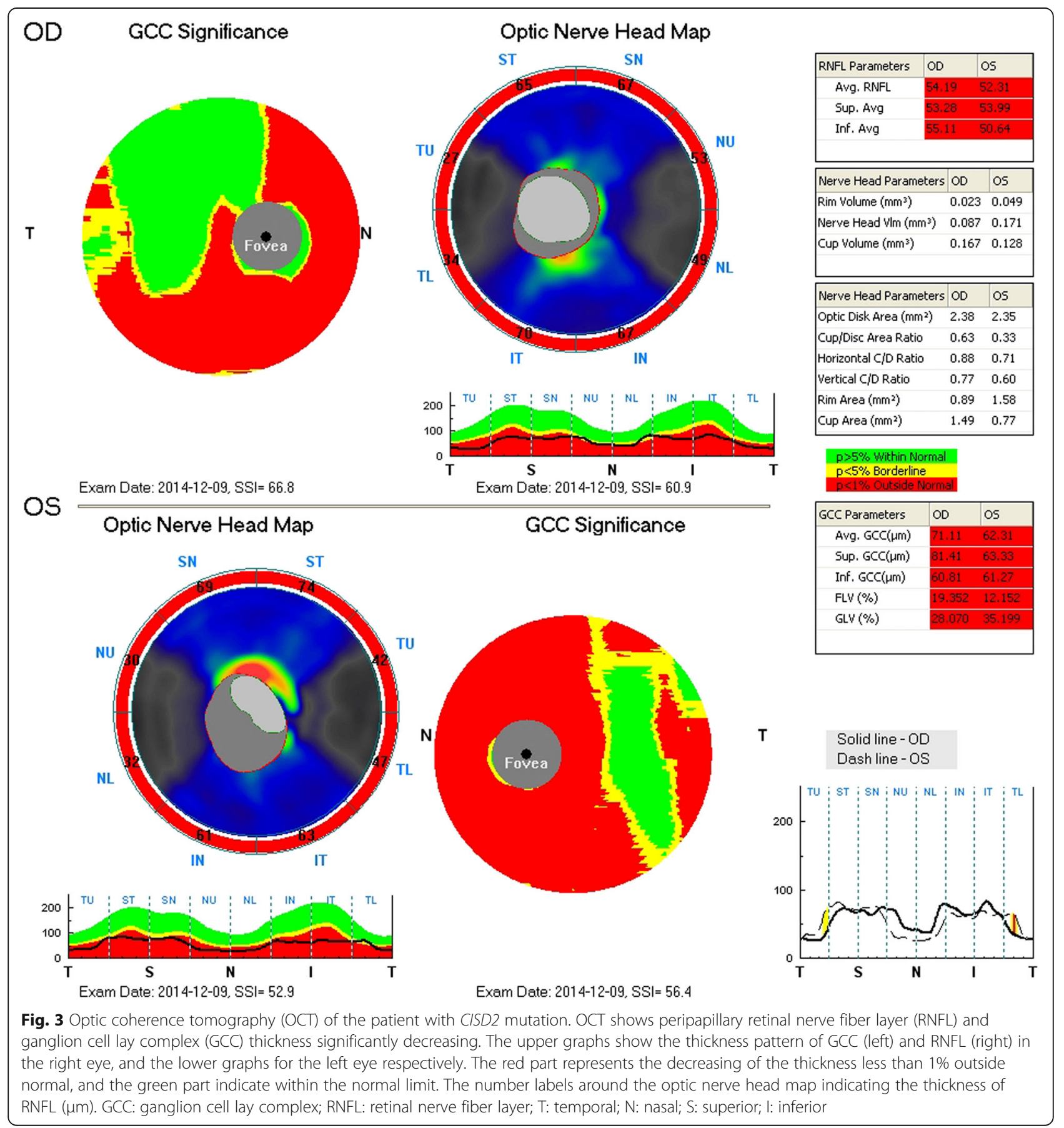


The detected mutations were checked in 110 Chinese normal controls by Sanger Sequence. Conservation of the mutation sites was evaluated by Clustal Omega [23]. Polymorphism Phenotyping 2 (PolyPhen2) [24] and Sorting Intolerant from Tolerant (SIFT) [25] were applied for the assessment of pathogenicity of detected mutations.

\section{Results}

\section{General clinical manifestations}

Four Chinese WFS patients from 4 different families were enrolled in our study. The demographic and clinical features of the 4 patients are shown in Table 1. All patients were male. The median age of patients was 25 years (range 11-42 years). Patient 1 came from consanguineous family and has an elder brother diagnosed of DM at age of 10 years and died from ketosis encephalopathy at age of 17 years. Patient 2 has an elder sister diagnosed of WFS with same symptoms.

All patients presented to our ophthalmology clinic because of progressive loss of vision (Table 1). They all had the coincidence of DM and OA. The median age at DM onset was 10 years (range 9-28 years). Three of them had various degree of hearing impairment: two patients had bilateral high-frequency hearing impairment and one had bilateral sensorineural deafness. Patient 1 was diagnosed as central diabetes insipidus by the water deprivation and desmopressin challenge test, and he also showed absence of the physiological high signal of the posterior pituitary gland on T1-weighted images of cranial MRI and an abnormal electroencephalography (EEG). No patient presented renal tract abnormalities, psychiatric diseases or behavioral disorders.

\section{Ophthalmologic manifestations}

In general, optic atrophy was observed in all four patients, demonstrated by the examination of fundus, MRI and OCT (Figs. 1, 2 and 3). The median age of presenting impaired vision and $\mathrm{OA}$ diagnosis was 12 years (range 7-39 years) and 17 years (range 7-42 years), respectively (Table 1). All patients presented severe vision loss and most of them had best corrected vision acuity
(BCVA) less than 20/400 (Table 1). All of them had normal pupillary responses. All patients presented color vision loss, especially patient 1 and patient 2 had all color vision defect. Perimetry examination demonstrated various types of vision field loss, presenting as central scotomas, constriction of peripheral visual filed, segmental arcuate defect or diffuse decreased sensitivity. OCT were abnormal in all patients, showing diffused thinning of peripapillary RNFL and macular ganglion cell lay complex (GCC) (Fig. 3). ERG were normal in all patients, VEP showed latency increase and amplitude reduction in P100 waves. No one had cataract and diabetic retinopathy.

\section{Genetic analysis}

Mutations in WFS1 or CISD2 gene were detected in all these patients, including one homozygous mutation on CISD2 and four missense mutations on WFS1 (Table 2). No other gene mutations or mitochondrial genome mutations were detected. Pedigrees with WFS in our study are shown in Fig. 4. For patient 1, we detected one novel frameshift mutation (p.Leu91fs) in exon 2 of CISD2 caused by the deletion of two nucleotides (c.272_273del). The homozygous mutations were inherited from his parents, who were first-cousin (Fig. 5). For patient 2, compound heterozygotic mutations (c.2020G > A+ c.1618 $\mathrm{T}>\mathrm{G}$ ) in WFS1 were identified and inherited from his father and mother respectively. The same mutations were also found in his sister, who had DM and OA as well (Additional file 1: Figure S1). Compound heterozygotic mutations in WFS1 were also detected in patient 3 (c.2020G > A+ c.1048 T > A) (Additional file 2: Figure S2). Patient 4 carried a de novo heterozygotic mutation $($ c. 937C $>\mathrm{T})$ in WFS1, which was absent in his parents (Additional file 3: Figure S3). Four variants, including c. $1618 \mathrm{~T}>\mathrm{G}, \mathrm{c} .1048 \mathrm{~T}>\mathrm{A}$, and c. $937 \mathrm{C}>\mathrm{T}$ in $W F S 1$ and c.272_273del in CISD2, were sequenced in 110 normal Chinese controls and none of mutations were detected.

Three of them were reported for the first time, including a frameshift mutation c.272_273del in CISD2 and two missense mutations c.1618 T > G, c.1048 T > A in WFS1. These mutations all locate in evolutionary conserved

Table 2 The mutations feature of patients with wolfram syndrome

\begin{tabular}{lllllllll}
\hline Case no. & Gene & $\begin{array}{l}\text { Nucleotide } \\
\text { changes }\end{array}$ & $\begin{array}{l}\text { Amino acid } \\
\text { changes }\end{array}$ & Type of mutation & Zygosity & References & PolyPhen2* $^{*}$ & SIFT** \\
\hline 1 & CISD2 & c.272_273del & p.Leu91fs & Frameshift & Homozygote & This study & - & Damaging \\
2 & WFS1 & c.1618T $>$ G & p.Trp540Gly & Missense & Compound heterozygote & This study & Possibly Damaging & Damaging \\
& & c.2020G $>$ A & p.Gly674Arg & Missense & & {$[26,27]$} & Probably Damaging & Damaging \\
3 & WFS1 & c.1048T $>$ A & p.Phe350lle & Missense & Compound heterozygote & This study & Probably Damaging & Damaging \\
& & c.2020G > A & p.Gly674Arg & Missense & & {$[26,27]$} & Probably Damaging & Damaging \\
4 & WFS1 & c.937C > T & p.His313Tyr & Missense & Heterozygote & {$[28-30]$} & Probably Damaging & Damaging \\
\hline
\end{tabular}

*PolyPhen2 Polymorphism Phenotyping 2, ** SIFT Sorting Intolerant from Tolerant. SIFT were used for the prediction of pathogenicity of all detected mutations and PolyPhen 2 were used for the prediction of pathogenicity of all missense mutations 

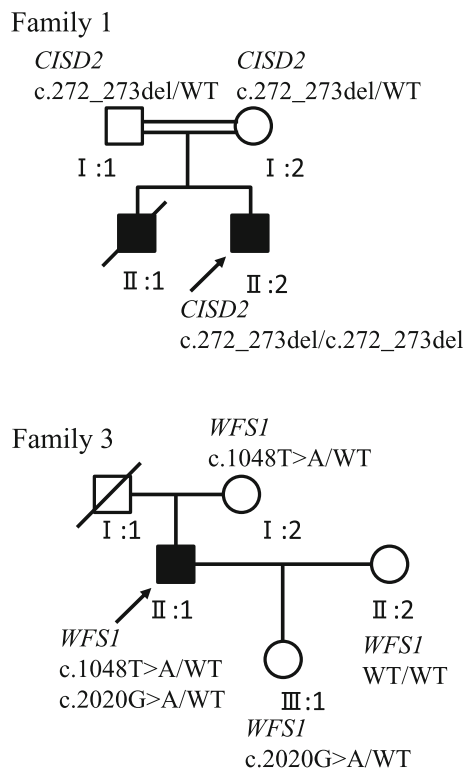

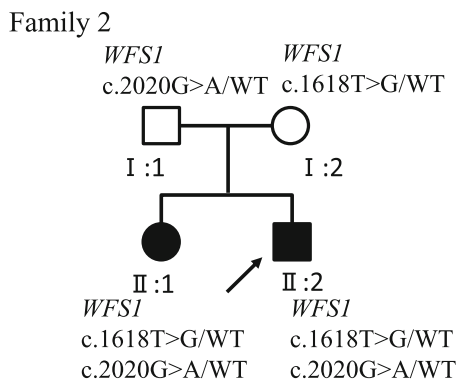

Family 4

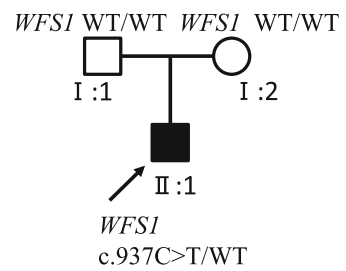

Fig. 4 Pedigrees of four WFS families. Black squares: affected males; black circles: affected females; white squares: unaffected males; white circles: unaffected females; arrow: the proband

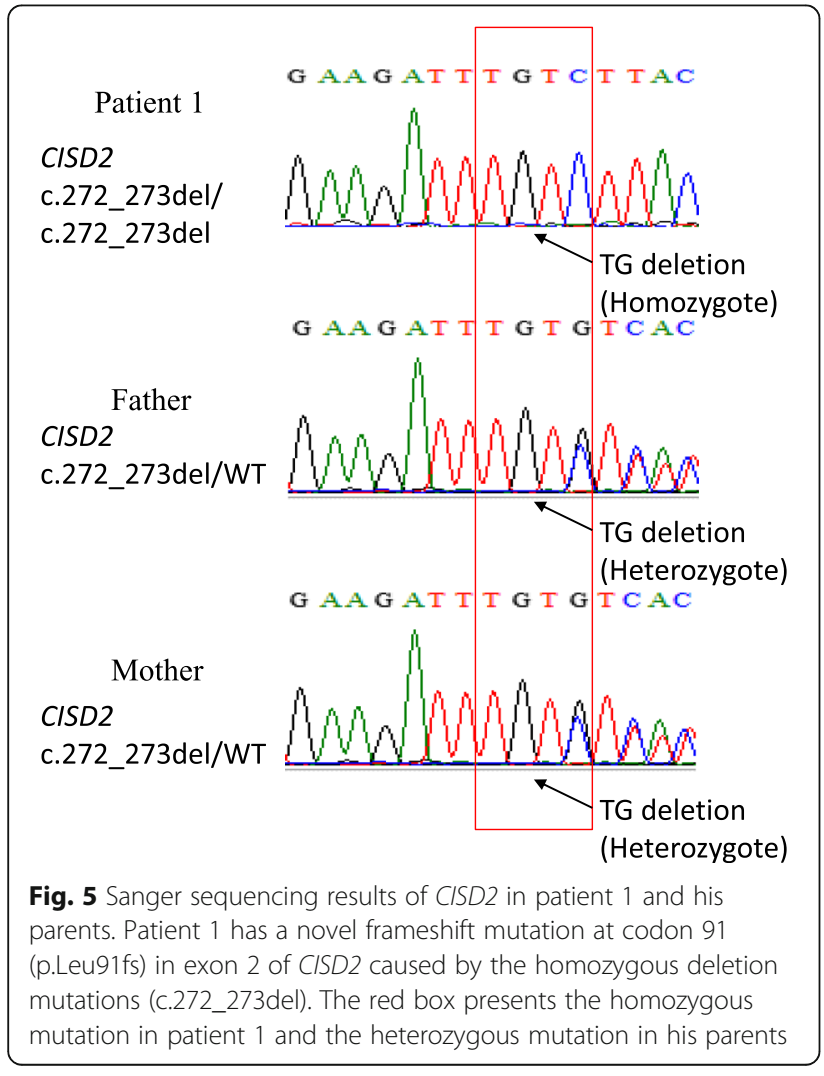

positions of CISD2 and wolframin by multiple sequence alignment across species (Additional file 4: Figure S4). Two novel missense mutations of WFS1 both located in exon 8 which encodes wolframin, a protein with nine predicted transmembrane domains and extracellular loops (Fig. 6). The novel variations of p.Trp540Gly (c.1618 T > G) and p.Phe350Ile (c.1048 T > A) in WFS1 and p.Leu91fs (c.272_273del) in CISD2 are all predicted to be highly deleterious by SIFT or PolyPhen2 (Table 2).

\section{Discussion}

In this study, we evaluated four Chinese WFS patients and descried their ophthalmologic characteristics, as well as reported three novel WFS1 and CISD2 mutations. Most patients presented at least three clinical manifestations and developed at least one in their first decade, which was consistent with the systematic review of WFS [5]. A wide range of ophthalmological findings were detected including severe vision acuity lost, declined color vision, constriction of visual fields and abnormal VEP, which were consistent with previous studies [31-33]. Notably, the presenting ages of impaired vision of some patients were early than OA diagnosis age, which suggested the insidiousness of vision loss in WFS. This indicates that ophthalmologist should be aware of the possibility of WFS in young patients with severe bilateral optic atrophy. Detailed medical history inquiry and appropriate genetic testing are highly recommended for these patients.

There are two genes, WFS1 and CISD2, were proven to cause WFS. CISD2 is a rare causative gene and 


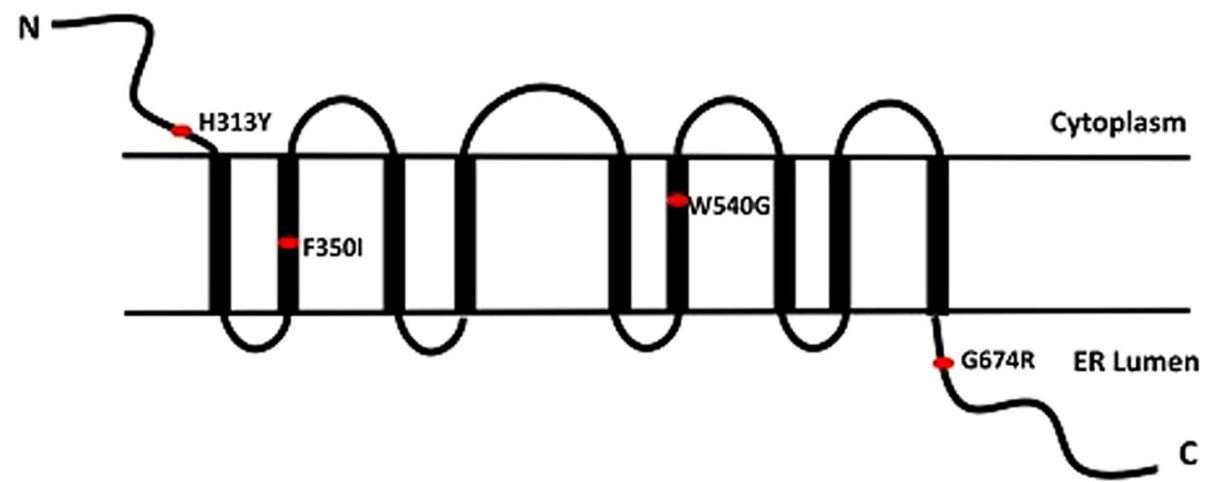

Fig. 6 The position of the mutations in WFS1. Predicted structure of the wolframin with nine putative transmembrane domains, and position of the mutations in WFS1 indicated by red cycles

autosomal-recessive mutations in CISD2 is the pathogeny of WFS2. So far, very limited mutations have been reported in this gene (Table 3) [17-20]. In our study, patient 1 was homozygous for the frame-shift mutation c.272_273del in CISD2, due to the parental consanguinity. This mutation was not detected in our Chinese control population. Patient 1 presented the most severe phenotype with rapid progression of disease and multisystem manifestations. The mutant CISD2 protein exerts a deleterious influence on ER-mitochondrial structure and function and ultimately participate in multisystem neurodegeneration [20]. WFS2 firstly was regarded as a subtype which has various unique features such as peptic ulcer and bleeding tendency [17-19]. In contrast, our patient presented classical features of WFS1, including early-onset DM, progressive OA, DI and neurodegenerative features. Haematological abnormalities and peptic ulcer has not been detected so far. Our study may support the point of view that WFS1 and WFS2, caused by different genes, has a continuous clinical spectrum [20]. Since this patient was still young, with the progression of WFS2, he may develop other signs of WFS2 in the future, so long term follow-up is needed.

Mutations in WFS1 gene are responsible for most WFS patients. Since the discovery of WFS1 in 1998, more than 300 different mutations have been identified in this gene [34] and majority of them located in the exon 8 encoding the nine transmembrane segments and the C-terminal tail of wolframin [33]. In this study, we found four missense mutations located in exon 8 of WFS1, two of them were first reported including c.1618 $\mathrm{T}>\mathrm{G}$ (p.Trp540Gly) and c.1048 T > A (p.Phe350Ile). The Sanger Sequence results in control population showed that these mutations are less likely to be polymorphisms. These two novel missense mutations are located in transmembrane domain. Multiple sequence alignment showed that they were positioned within evolutionary conserved regions of wolframin. And they were predicted to be deleterious by different tools (Table 2). Notably, the mutation c.2020G >A was found in two unrelated patients in our study. This mutation was previously reported in 4 patients with DM and OA without DI and deafness [26, 27]. The allele frequency of $\mathrm{A}$ is < 0.0001 in Han Chinese by the 1000 Genomes Project [35]. Our result indicates that this mutation is probably a hotspot in Chinese WFS patients, which needs to be verified by more cases. Only one heterozygous mutation (c.937C > T, p.His313Tyr) was found in patient 4, which was previously detected in three patients with OA, very early DM diagnosis and profound hearing loss [28-30]. Coincidentally, patient 4 was diagnosed hearing loss much earlier than OA, which might provide an evidence that this mutation cause more hearing impairment than visual disability.

\section{Conclusions}

Our study showed a group of Chinese patients with WFS who had various clinical features. Genetic analysis

Table 3 CISD2 mutations reported in patients with Wolfram Syndrome type 2

\begin{tabular}{llllllll}
\hline Gene & Population & Nucleotide changes & Amino acid changes & Exon & Consequences & Zygosity & References \\
\hline CISD2 & Jordanian & c.109G $>$ C & p.Glu37Gln & Exon 2 & Missense mutation, affects mRNA splicing & Homozygote & [19] \\
CISD2 & Caucasian & intragenic deletion & - & Exon 2 & Exon 2 deletion & Homozygote & [18] \\
CISD2 & Italian & c.103 +1G $>$ A & - & Intron 1 & Exon 1 be skipped & Homozygote & [17] \\
CISD2 & Moroccan & c.215A $>$ G & p.Asn72Ser & Exon 2 & Missense mutation & Homozygote & [20] \\
CISD2 & Chinese & c.272_273del & p.Leu91fs & Exon 2 & Frameshift mutation & Homozygote & This study \\
\hline
\end{tabular}


detected three novel mutations in WFS1 and CISD2. This is the first report of Chinese patient with WFS2. Our study also illustrates the complexity and heterogeneity of WFS. So genetic testing is recommended for clinical optic nerve atrophy patients with highly suspected WFS, especially when diabetes mellitus is concomitant.

\section{Additional files}

Additional file 1: Figure S1. Electropherograms of identified mutations in patient 2 and his families. (JPG $139 \mathrm{~kb}$ )

Additional file 2: Figure S2. Electropherograms of identified mutations in patient 3 and his families (his father has passed away). (JPG $133 \mathrm{~kb}$ )

Additional file 3: Figure S3. Electropherograms of identified mutations in patient 4 and his families. (JPG $56 \mathrm{~kb}$ )

Additional file 4: Figure S4. Multiple alignment of amino acid sequences of WFS1 and CISD2 across species. (JPG $273 \mathrm{~kb}$ )

\section{Abbreviations}

BCVA: Best corrected vision acuity; CISD2: CDGSH iron-sulfur domain-containing protein 2; CT: Computed tomography; D: Deafness; Dl: Diabetes insipidus; DM: Diabetes mellitus; EEG: Electroencephalography; ER: Endoplasmic reticulum; ERG: Electroretinography; ERISP: Encodes endoplasmic reticulum intermembrane small protein; GCC: Ganglion cell lay complex; MRI: Magnetic resonance imaging; NGS: Next generation sequence; OA: Optic atrophy; OCT: Optic coherence tomography; OD: Right eye; OS: Left eye; PolyPhen2: Polymorphism Phenotyping 2; RNFL: Retinal nerve fiber layer: SIFT: Sorting Intolerant from Tolerant; VA: Visual acuity; VEP: Visual evoked potentials; WFS: Wolfram Syndrome; WFS1: Wolfram Syndrome type 1; WFS2: Wolfram Syndrome type 2

\section{Acknowledgements}

We are grateful to the biobank of the Eye Ear Nose and Throat Hospital of Fudan University. We would also like to thank all the patients and their families.

\section{Authors' contributions}

T-GH and K-XM collected the clinical samples and carried out ophthalmologic examination. Z-YJ, C-YH and F-LL analyzed sequencing results and drafted the manuscript. Z-YJ, C-YH and T-GH revised the manuscript. W-JH participated in the management of the patients. All authors read and approved the final manuscript.

\section{Funding}

This work was supported by funds from the National Science Foundation of China [No. 81570887].

\section{Availability of data and materials}

The datasets supporting the conclusions of this article are included within the article.

\section{Ethics approval and consent to participate}

This study was approved by the Eye Ear Nose and Throat Hospital of Fudan University Institutional Review Board.

\section{Consent for publication}

Written informed consent was obtained from the patients or their legal guardians.

\section{Competing interests}

The authors declare that they have no competing interests.

\section{Author details}

'Department of Ophthalmology and Visual Science, Eye, Ear, Nose and Throat Hospital, Shanghai Medical College, Fudan University, Shanghai,
China, 83 Fenyang Road, Shanghai 200031, China. ${ }^{2}$ State Key Laboratory of Medical Neurobiology, Institutes of Brain Science and Collaborative Innovation Center for Brain Science, Eye Ear Nose and Throat Hospital of Fudan University, 83 Fenyang Road, Shanghai 200031, China. ${ }^{3}$ NHC Key Laboratory of Myopia (Fudan University), Laboratory of Myopia, Chinese Academy of Medical Sciences, Eye Ear Nose and Throat Hospital of Fudan University, 83 Fenyang Road, Shanghai 200031, China. ${ }^{4}$ Shanghai Key Laboratory of Visual Impairment and Restoration (Fudan University), Eye Ear Nose and Throat Hospital of Fudan University, Shanghai, China, 83 Fenyang Road, Shanghai 200031, China.

Received: 4 February 2019 Accepted: 19 July 2019

Published online: 07 August 2019

\section{References}

1. Barrett TG, Bundey SE, Macleod AF. Neurodegeneration and diabetes: UK nationwide study of Wolfram (DIDMOAD) syndrome. Lancet. 1995;346:1458-63.

2. Matsunaga $\mathrm{K}$, et al. Wolfram syndrome in the Japanese population; molecular analysis of WFS1 gene and characterization of clinical features. PLoS One. 2014;9:e106906.

3. Barrett TG, Bundey SE. Wolfram (DIDMOAD) syndrome. J Med Genet. 1997; 34:838-41.

4. Pilley SF, Thompson HS. Familial syndrome of diabetes insipidus, diabetes mellitus, optic atrophy, and deafness (didmoad) in childhood. $\mathrm{Br} J$ Ophthalmol. 1976;60:294-8.

5. de Heredia ML, Cleries R, Nunes V. Genotypic classification of patients with Wolfram syndrome: insights into the natural history of the disease and correlation with phenotype. Genet Med. 2013;15:497-506.

6. The EURO-WABB Project. http://euro-wabb.org/. Accessed 3 June 2019.

7. Farmer A, et al. EURO-WABB: an EU rare diseases registry for Wolfram syndrome, Alstrom syndrome and Bardet-Biedl syndrome. BMC Pediatr. 2013;13:130

8. Inoue $\mathrm{H}$, et al. A gene encoding a transmembrane protein is mutated in patients with diabetes mellitus and optic atrophy (Wolfram syndrome). Nat Genet. 1998:20:143-8.

9. Khanim F, Kirk J, Latif F, Barrett TG. WFS1/Wolframin mutations, Wolfram syndrome, and associated diseases. Hum Mutat. 2001:17:357-67.

10. Takeda K, et al. WFS1 (Wolfram syndrome 1) gene product: predominant subcellular localization to endoplasmic reticulum in cultured cells and neuronal expression in rat brain. Hum Mol Genet. 2001;10:477-84.

11. Hofmann S, Philbrook C, Gerbitz KD, Bauer MF. Wolfram syndrome: structural and functional analyses of mutant and wild-type wolframin, the WFS1 gene product. Hum Mol Genet. 2003;12:2003-12.

12. Yamamoto $\mathrm{H}$, et al. Wolfram syndrome 1 (WFS1) protein expression in retinal ganglion cells and optic nerve glia of the cynomolgus monkey. Exp Eye Res. 2006;83:1303-6.

13. Schmidt-Kastner $R$, et al. Expression of the diabetes risk gene wolframin (WFS1) in the human retina. Exp Eye Res. 2009;89:568-74

14. Fonseca $S G$, et al. Wolfram syndrome 1 gene negatively regulates ER stress signaling in rodent and human cells. J Clin Invest. 2010;120:744-55.

15. Rigoli L, Lombardo F, Di Bella C. Wolfram syndrome and WFS1 gene. Clin Genet. 2011;79:103-17.

16. Lu S, et al. A calcium-dependent protease as a potential therapeutic target for Wolfram syndrome. Proc Natl Acad Sci U S A. 2014;111:E5292-301.

17. Rondinelli M, Novara F, Calcaterra V, Zuffardi O, Genovese S. Wolfram syndrome 2: a novel CISD2 mutation identified in Italian siblings. Acta Diabetol. 2015;52:175-8.

18. Mozzillo $\mathrm{E}$, et al. A novel CISD2 intragenic deletion, optic neuropathy and platelet aggregation defect in Wolfram syndrome type 2. Bmc Med Genet. 2014;15:88.

19. Amr S, et al. A homozygous mutation in a novel zinc-finger protein, ERIS, is responsible for Wolfram syndrome 2. Am J Hum Genet. 2007;81:673-83.

20. Rouzier C, et al. A novel CISD2 mutation associated with a classical Wolfram syndrome phenotype alters $\mathrm{Ca}^{2+}$ homeostasis and ER-mitochondria interactions (vol 26, pg 1599, 2017). Hum Mol Genet. 2017;26:1786.

21. Wang $\mathrm{CH}$, et al. Cisd2 modulates the differentiation and functioning of adipocytes by regulating intracellular $\mathrm{Ca}^{2+}$ homeostasis. Hum Mol Genet. 2014;23:4770-85.

22. Alberti KG, Zimmet PZ. Definition, diagnosis and classification of diabetes mellitus and its complications. Part 1: diagnosis and classification of 
diabetes mellitus provisional report of a WHO consultation. Diabet Med. 1998;15:539-53.

23. Sievers F, et al. Fast, scalable generation of high-quality protein multiple sequence alignments using Clustal omega. Mol Syst Biol. 2011;7:539.

24. Adzhubei IA, et al. A method and server for predicting damaging missense mutations. Nat Methods. 2010;7:248-9.

25. Kumar P, Henikoff S, Ng PC. Predicting the effects of coding nonsynonymous variants on protein function using the SIFT algorithm. Nat Protoc. 2009:4:1073-82.

26. Gomez-Zaera M, et al. Presence of a major WFS1 mutation in Spanish Wolfram syndrome pedigrees. Mol Genet Metab. 2001;72:72-81.

27. Aloi C, et al. Wolfram syndrome: new mutations, different phenotype. PLoS One. 2012;7:e29150.

28. Marshall BA, et al. Phenotypic characteristics of early Wolfram syndrome. Orphanet J Rare Dis. 2013;8:64

29. Hansen $L$, et al. Mutation analysis of the WFS1 gene in seven Danish Wolfram syndrome families; four new mutations identified. Eur J Hum Genet. 2005;13:1275-84.

30. De Franco E, et al. Dominant ER stress-inducing WFS1 mutations underlie a genetic syndrome of neonatal/infancy-onset diabetes, congenital sensorineural deafness, and congenital cataracts. Diabetes. 2017;66:2044-53.

31. Soares A, et al. Ophthalmologic manifestations of Wolfram syndrome: report of 14 cases. Ophthalmologica. 2018:1-4.

32. Zmyslowska A, et al. Retinal thickness as a marker of disease progression in longitudinal observation of patients with Wolfram syndrome. Acta Diabetol. 2017:54:1019-24.

33. Grenier J, et al. WFS1 in optic neuropathies: mutation findings in nonsyndromic optic atrophy and assessment of clinical severity. Ophthalmology. 2016;123:1989-98.

34. Bansal V, Boehm BO, Darvasi A. Identification of a missense variant in the WFS1 gene that causes a mild form of Wolfram syndrome and is associated with risk for type 2 diabetes in Ashkenazi Jewish individuals. Diabetologia. 2018:61:2180-8

35. 1000 Genomes Project. https:/www.ncbi.nlm.nih.gov/variation/tools/1 000genomes/?gts=rs200672755. Accessed 3 February 2019.

\section{Publisher's Note}

Springer Nature remains neutral with regard to jurisdictional claims in published maps and institutional affiliations.

Ready to submit your research? Choose BMC and benefit from:

- fast, convenient online submission

- thorough peer review by experienced researchers in your field

- rapid publication on acceptance

- support for research data, including large and complex data types

- gold Open Access which fosters wider collaboration and increased citations

- maximum visibility for your research: over $100 \mathrm{M}$ website views per year

At $\mathrm{BMC}$, research is always in progress.

Learn more biomedcentral.com/submissions 